\title{
The use of a quadcopter-mounted hyper-spectral spectrometer for examining reflectance in Scottish coastal waters.
}

\author{
Rebecca J. Weeks, Philip Anderson, Keith Davidson \\ PhD Candidate, Director of Studies, Co-Supervisor respectively \\ Technology/Molecular and Microbial Biology \\ SAMS \\ Oban, Scotland, UK \\ Email: rjweeks90@gmail.com
}

\author{
David McKee \\ Co-Supervisor \\ Physics \\ University of Strathclyde \\ Glasgow, Scotland, UK
}

\begin{abstract}
Remote sensing of the marine coastal environment is useful for obtaining information about processes occurring within it. Monitoring has traditionally been carried out in situ, before investment increased in remote techniques such as manned planes and satellites. This paper proposes the use of remotely piloted aircraft systems (RPAS) as an alternative methodology, with an aim of increasing the spectral, spatial and temporal resolution of data whilst reducing the associated costs and risks. A novel 'spectro-copter' system, comprising of an integrated dual field-of-view, miniaturised, hyper-spectral spectrometer aboard a purpose-built quadcopter is presented, developed at the Scottish Association for Marine Science (SAMS), Oban. This has been produced with a view to investigating reflectance from Scottish coastal waters, which can give inferences as to the concentrations of various constituents present $[1,2]$. Initial test flights show the 'spectro-copter' system is capable of flights of $\sim 20$ minutes, sufficient for meaningful data collection, despite late adjustments incurring increased weight and an associated reduction in efficiency. Early results demonstrate that the setup is capable of discerning differences in $R$ at a high spectral resolution. Further work is ongoing in order to assess the capacity for establishing this equipment as a routine technique for the monitoring of coastal harmful algal blooms (HAB), which currently relies upon physical sampling in combination with satellites. $\mathrm{HAB}$ events are noted to be increasing in severity and frequency [3, 4], with knock-on health and economic impacts, particularly for the rising aquaculture industry in Scottish coastal waters [5]. Therefore advancement of an alternative, affordable monitoring technique would be extremely beneficial.
\end{abstract}

Keywords-remote-sensing; reflectance; remotely piloted aircraft system; quadcopter; hyper-spectral; spectrometer; Scottish coastal waters.

\section{INTRODUCTION}

This paper seeks to present a purpose-built remotely piloted aircraft system (RPAS) in combination with a customdeveloped hyper-spectral spectrometer, designed for the purpose of remotely examining Scottish coastal water reflectance $(R)$. Physical water sampling is a technique traditionally utilised to gain information about the components within a given area of water. However, information may also be inferred from $R$ results. These can change in a predictable way in response to certain constituents and their concentrations [1]. $R$ represents the ratio, as a percentage, between upwelling irradiance data from a given target of interest and downwelling irradiance from the sun.

The way in which light propagates through a medium is described using radiative transfer theory and depends on its composition. Optically significant constituents within the marine environment comprise of both dissolved and particulate matter, with particulates including both biological and physical sources. The optical properties of an area of water can allow for the calculation of further properties, e.g. chlorophyll $a$ concentration, which can be used as a proxy for identification and quantification of certain species of phytoplankton.

The coastal marine ecosystem can be difficult and expensive to examine. With regards to ocean colour remote sensing, efforts have typically focussed on manned plane and satellite monitoring techniques. Manned planes are expensive, leading to infrequent sampling and poor temporal resolution $[6,7]$. They may also be restricted in their extent geographically, for example due to adjacent land. Additionally, there is an associated risk to human life [8], therefore flying locations and weather conditions for such work are restricted.

Satellite monitoring is one remote sensing alternative which avoids these risks, however, this method is limited in its spatial and temporal resolution, with so-called high resolution satellites such as Landsat and MODIS presenting a pixel size of $\sim 30 \mathrm{~m}$ and $\sim 1 \mathrm{~km}$ respectively [6]. Spatial and spectral resolutions globally are generally insufficient for discerning fine level details e.g. iceberg location and orientation [9]. Establishment of a new satellite is costly and payloads cannot be easily altered, maintained or replaced [10]. In addition, notable for Scottish waters, datasets frequently suffer areas of missing information due to cloud cover [5].

Remote sensing techniques which utilise remotely piloted technology are becoming widely recognised as a viable alternative to those outlined above. The use of RPAS, such as 'copter and fixed wing planes, as an alternative to satellite monitoring would successfully eradicate data loss due to cloud presence since they operate at much lower altitudes, which also allows increased resolution. In addition, this removes the complications associated with selection and application of a suitable atmospheric correction [11].

\section{EQUIPMENT DEVELOPMENT}

\section{A. Spectrometer Development}

The spectrometer system presented here, since marketed as the 'Piccolo', was developed by the Field Spectroscopy Facility (FSF), University of Edinburgh, in conjunction with R. Weeks 
and Dr P. Anderson (SAMS). Reference [12] details the early stages of this dual field-of-view system. The configuration presented here utilises an Ocean Optics USB2000+ spectrometer, with an operational spectral range from 400 to $850 \mathrm{~nm}$, with an optical slit width of $100 \mu \mathrm{m}$. This larger-thantypical slit width was selected due to the comparatively low reflectance of the ocean in comparison with land-based targets. The bi-directional system uses a bifurcated fibre optic pair to obtain near-simultaneous downwelling irradiance and upwelling radiance data, in order to account for changing irradiance conditions. Alternative RPAS-mounted systems presented in the literature typically include only one field-ofview, such as the Ocean Optics STS-spectrometer [13], although one published example presents a dual system onboard a fixed wing aircraft [14]. Due to the use of a fixed wing platform, the resolution of this system is limited to $30 \mathrm{~m}$, with a minimum flight speed required to prevent stalling.

Data is collected in four channels, controlled by two shutters. The system records a dark signal, with both shutters closed. The downwelling shutter then opens and photons are recorded for the integration time set manually. Following this reading, the first shutter closes whilst the other opens to record a corresponding upwelling measurement before both shutters close once more for a second dark signal reading.

Processing of raw spectrometer data follows a five step procedure, outlined here:

1. Subtract the corresponding dark signal from the downand upwelling channels.

2. Divide both channels by their respective integration times.

3. Convert pixel numbers to wavelength (nm) and re-grid each channel onto the same spectral scale.

4. Divide the upwelling counts by the corresponding downwelling counts to calculate raw reflectance $(R)$.

5. Calculate corrected $R$ : apply the appropriate correction factor for the given sky conditions, obtained from the nominal 20\% reflectance panel (explained below).

Radiometric calibration was not completed since the system may need to be disassembled and reassembled for use in various platforms or for transport between field sites. Since the system was not absolutely calibrated, it was necessary to apply a correction factor to raw $R$ data. Spectra were recorded above a calibrated, nominal $20 \%$ reflectance panel prior to any field measurements in order to calculate a correction factor per wavelength. Following this procedure, the calculation provides $R$ as a reflectance ratio (\%) at each wavelength of interest. It was found that this correction factor varied according to the given sky conditions, i.e. with varying cloud cover. This indicates that the plate is non-lambertian, counter to initial reports, discussed below.

The Piccolo system was further characterised and calibrated at SAMS. Following testing, the field of view was found to be slightly oval in shape, averaging $26.5^{\circ}$. The system has a reported full-width half maximum of $3.96 \mathrm{~nm}$. However, laboratory tests showed this value to be higher on average, increasing with wavelength, and giving a higher resolution for the upwelling channel versus the downwelling channel.

\section{B. 'Copter Development}

RPAS are extremely useful for detailed, small-scale studies of coastal areas. These are powered vehicles that utilise aerodynamic forces to produce lift, capable of autonomous flight or remote piloting to transport payloads [10]. They are cheap, presenting a comparatively disposable option for highrisk locations and weather conditions $[6,10]$. This lends them to surveys operating from boat platforms.

Due to the need for an affordable, suitably sized RPAS system, a custom 'copter was developed. Commercially available alternatives at the time suffered a significant gap, with small systems (lift capacity: $500 \mathrm{~g}$ ) jumping straight to more costly, large-footprint 'copters (up to $7 \mathrm{~kg}$ ). The design had to be small: firstly for ease of transportation to remote field sites, but additionally to allow take-off and landing in restricted areas. A 'copter system was selected due to its capacity for low flying speeds, whereas a fixed wing alternative would stall.

The finished system incorporated a PixHawk flight controller, running Arducopter v3, to allow fully piloted, and both semiand fully autonomous missions. Flight data included time, date, GPS position, altitude, pitch, roll and yaw. This allowed synchronisation with data from the on-board hyper-spectral spectrometer. The final design carefully considered the compromise between practical size, operational thrust and battery capacity/weight to allow maximum flight time between battery changes given the weight of the finalised payload.

\section{Spectrometer Integration}

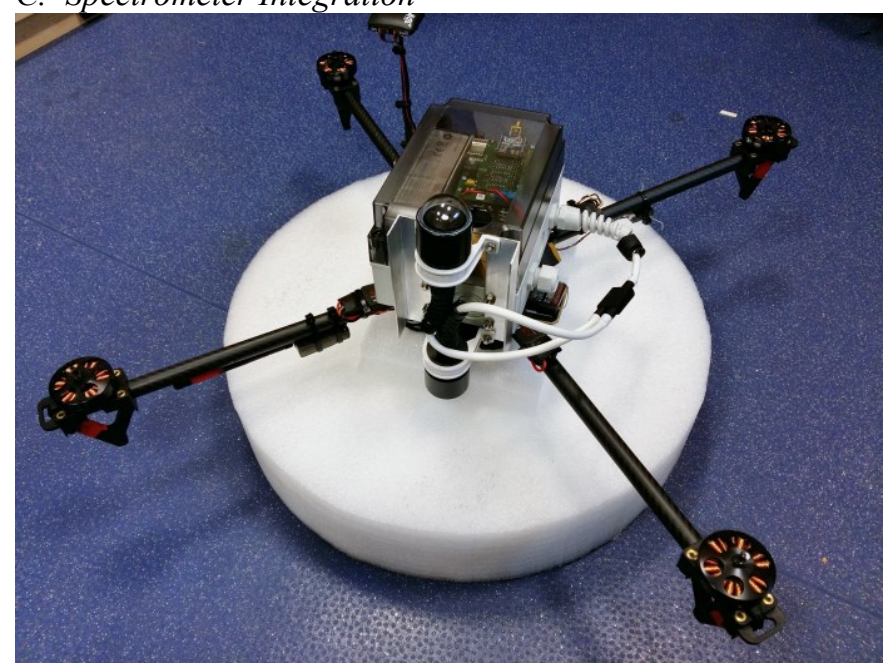

Fig. 1: Image displaying the completed integration of the IP68 housed, dual field-of-view spectrometer aboard the custom-built quadcopter system (rotary blades not included). The white polystyrene circle is for display purposes only and not part of the flying setup.

The complete setup is displayed in fig. 1. In order to operate safely within the marine environment, IP68 protective housing was added prior to the Piccolo's integration onto the quadcopter to form the 'spectro-copter'. This increased the system's total weight from roughly $900 \mathrm{~g}$ to $1200 \mathrm{~g}$, taking the total system weight to $3340 \mathrm{~g}$, which incurred an associated decrease in endurance. During initial test flights it was also decided that the addition of a GoPro Hero 4 camera to the system would be invaluable. This would provide 
corresponding visual information to support spectrometer data recorded during the equipment's development phase. However, with this came an additional 240g, increasing the current consumption during flights by approximately $25 \%$ and reducing the available total flight time to $\sim 20$ minutes.

\section{LAND-BASED TESTS: RESULTS AND DisCUSSION}

Inter-comparison of data obtained using different systems via simultaneous measurements under almost identical conditions may indicate their consistency and related uncertainties [2]. In order to minimise the risks associated with both flying and operation over water, Piccolo data were first collected over a number of land-based targets alongside a pair of radiometrically calibrated Trios Ramses hyper-spectral radiometers (ACC2-VIS, recording downwelling irradiance and ARC-VIS, recording upwelling radiance). This aimed to test the suitability of the data processing technique detailed in section IIA., whilst also avoiding the complications associated with surface reflectance, a major factor to consider for abovewater measurements.

Data collection was carried out over a variety of targets on $21^{\text {st }}$ March 2016 under 100\% cloud cover, with no precipitation. Ambient conditions were stable for the duration of each target measurement, which was carried out for the equivalent time of 25 Piccolo spectra before an average was taken for increased reliability. Results are shown in fig. 2. As can be seen, the overall shape of $R$ results from both sets of equipment appears almost identical. However, the magnitude of each spectrum varies quite dramatically between setups, particularly in the near-infrared for natural substrates such as grass and seaweed. This difference is quantified in table 1 , with mean difference across the wavelengths 400 to $850 \mathrm{~nm}$ varying from 39.16 to $50.90 \%$.

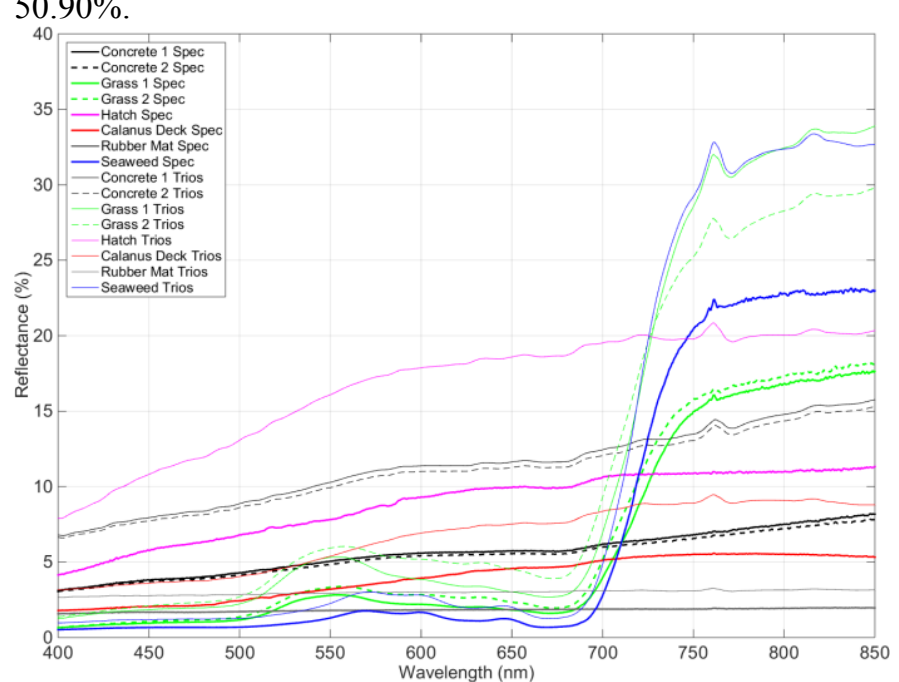

Fig. 2: Comparison between $R$ values (\%) obtained using the spectro-copter and the radiometrically calibrated Trios Ramses radiometer setups.

A factor of pi was used to convert Ramses radiance results to irradiance values prior to $R$ calculations for direct comparison with the Piccolo results. This assumes each target to be lambertian, reflecting radiance equally into all directions [15]. The targets displayed in fig. 2 may not fulfil this assumption, which could explain the difference in absolute $R$ values.
During the course of developing a suitable data processing technique, it was also noted that the calibrated nominal $20 \%$ reflectance panel was not lambertian, counter to initial reports. Blanket cloud was found to produce the most stable correction factor (compared to sunny conditions), with an average for such conditions calculated and applied to produce fig. 2 . However, it is possible that this may not perform as expected from one measurement to the next. It would have been highly beneficial to record the reflectance of the panel with both spectrometer setups. However, due to borrowing equipment and personnel from different institutes, the Ramses radiometers and panel were not available on coinciding dates.

\begin{tabular}{|l|l|l|l|}
\hline Target & $\begin{array}{l}\text { Min Diff } \\
(\%)\end{array}$ & Max Diff (\%) & Mean Diff (\%) \\
\hline Concrete (1) & 47.48 & 54.04 & 50.73 \\
\hline Concrete (2) & 48.53 & 54.17 & 50.90 \\
\hline Grass (1) & 38.64 & 50.57 & 45.92 \\
\hline Grass (2) & 36.88 & 52.07 & 44.61 \\
\hline Metal Hatch & 44.04 & 51.65 & 46.94 \\
\hline $\begin{array}{l}R V \quad \text { Calanus } \\
\text { Painted Deck }\end{array}$ & 37.64 & 44.05 & 40.40 \\
\hline Rubber Mat & 36.77 & 44.05 & 39.16 \\
\hline Seaweed & 28.27 & 49.72 & 39.59 \\
\hline
\end{tabular}

Table 1: Minimum, maximum and mean percentage differences per target using the two instrument setups.

In general, reference panels utilised for this technique tend to be highly reflective $(>90 \%)$ and reportedly lambertian in nature. However, due to the large optical slit $(100 \mu \mathrm{m})$ used in the Piccolo system it was only possible to use a nominal $20 \%$ reflectance panel, in order to avoid saturation of the system. It is thought that increased doping with grey pigment may have an associated impact on its lambertian nature [16]. This suggests that such a method may not be appropriate for reliable correction of $R$ data without first characterising the reference panel with regards to its bi-directional reflectance distribution factor (BRDF), or non-lambertian, nature.

\section{PROPOSED APPLICATIONS}

A proposed target for RPAS remote-sensing technology is coastal harmful algal blooms (HABS), documented in Scottish waters $[3,4]$. Some species are noted to have negative impacts on aquaculture. Problematic species include those producing toxins, e.g. Alexandrium and Pseudo-nitzschia [5], as well as species which bloom to negative concentrations. This can create various issues, from nutrient depletion, to creation of anoxia during their breakdown, to fatty acid production destroying red blood cells, e.g. by Karenia mikimotoi [17]. Movement by wild fish populations minimises the effects of such blooms, however the captive nature of fish farms prevents escape, resulting in devastating mortality events [18]. The frequency of such events is predicted to increase further in the future as a result of eutrophication and climate change $[3,4]$. This will have augmented health impacts on both human and marine life, as well as economic effects, therefore the development of a comparatively cheap and simple monitoring technique would be invaluable to the aquaculture industry. Recently, remote sensing of blooms via satellite imagery has been gaining momentum. This early warning 
technique relies on chlorophyll $a$ detection as a proxy for phytoplankton bloom presence [5]. However, issues mentioned above, e.g. infrequent pass rates and cloud cover means such techniques are not likely to be appropriate for rapid identification and subsequent management of HABs in Scottish coastal waters. Regular RPAS surveys, on the other hand, have the potential to overcome such issues.

A key next step is the presentation of data collected using the spectro-copter setup over water. This brings associated issues of sun and sky reflectance at the water surface, which must be corrected for before meaningful data may be obtained. The inaccuracies associated with correcting $R$ using the nonlambertian plate must also be dealt with, although it is possible to carry out a vicarious calibration using the paired Ramses radiometer setup.

\section{CONCLUSIONS}

RPAS are significantly more affordable and accessible than existing techniques, with the capacity for recording higher spatial, temporal and spectral resolution data of a given area of interest. This, along with their capability for increasing safety in hazardous locations and weather conditions makes them an obvious choice of technology for future investment. Not only could the technology be employed for HAB monitoring, but $R$ signals could potentially be utilised for multiple ecosystem health and functioning observations. This could allow the supplementation of satellite techniques for a variety of targets: from the mapping of jellyfish or macro-algal blooms, to submerged vegetation and seagrasses [19], surface monitoring of oil spills, and even sea ice.

\section{ACKNOWLEDGMENTS}

R.J. Weeks would like to thank her PhD supervisors for their guidance throughout, with information in this paper taken from her PhD thesis. She thanks SAMS engineer Shane Rodwell, quadcopter developer and pilot, as well as Alasdair MacArthur and Iain Robinson (FSF) for their development of the spectrometer system.

\section{REFERENCES}

[1] 1. Mobley, C.D., Handbook of Optics. 1994.

[2] 2. Zibordi, G., et al., In situ determination of the remote sensing reflectance: an inter-comparison. Ocean Science, 2012. 8(4): p. 567-586.

[3] 3. Anderson, D.M., A.D. Cembella, and G.M. Hallegraeff, Progress in Understanding Harmful Algal Blooms: Paradigm Shifts and New Technologies for Research, Monitoring, and Management. Annual Review of Marine Science, 2012. 4(1): p. 143-176.

[4] 4. Carvalho, G.A., et al., Satellite remote sensing of harmful algal blooms: A new multi-algorithm method for detecting the Florida Red Tide (Karenia brevis). Harmful Algae, 2010. 9(5): p. 440-448.

[5] 5. Davidson, K., et al., A large and prolonged bloom of Karenia mikimotoi in Scottish waters in 2006. Harmful Algae, 2009. 8(2): p. 349-361.
[6] 6. Anderson, K. and K.J. Gaston, Lightweight unmanned aerial vehicles will revolutionize spatial ecology. Frontiers in Ecology and the Environment, 2013. 11(3): p. 138-146.

[7] 7. Mitchell, C., A. Cunningham, and D. McKee, Remote sensing of shelf sea optical properties: Evaluation of a quasi-analytical approach for the Irish Sea. Remote Sensing of Environment, 2014. 143: p. 142153.

[8] 8. Ogden, L.E., Drone Ecology. BioScience, 2013. 63(9): p. 776-776.

[9] 9. McGill, P.R., et al., Aerial surveys and tagging of free-drifting icebergs using an unmanned aerial vehicle $(U A V)$. Deep Sea Research Part II: Topical Studies in Oceanography, 2011. 58(11-12): p. 13181326.

[10] 10. Brears, R., Using unmanned aerial vehicles in Antarctica. anta.canterbury.ac.nz, 2011.

[11] 11. Blondeau-Patissier, D., et al., A review of ocean color remote sensing methods and statistical techniques for the detection, mapping and analysis of phytoplankton blooms in coastal and open oceans. Progress in Oceanography, 2014.

[12] 12. MacArthur, A., et al., A dual-field-of-view spectrometer system for reflectance and fluorescence measurements (Piccolo Doppio) and correction of etaloning. The Fifth International Workshop on Remote Sensing of Vegetation Fluorescence, 2014.

[13] 13. Von Bueren, S., et al., Deploying four optical UAVbased sensors over grassland: challenges and limitations. Biogeosciences, 2015. 12(1): p. 163.

[14] 14. Shang, S., et al., Sensing an intense phytoplankton bloom in the western Taiwan Strait from radiometric measurements on a UAV. Remote Sensing of Environment, 2017. 198: p. 85-94.

[15] 15. Mobley, C.D., E. Boss, and C.S. Roesler, Ocean Optics Web Book. 2014.

[16] 16. Yuen, J.D., Small Scale Unmanned Aircraft for Environmental Monitoring, in Department of Mechanical Engineering. 2014, University of Alberta.

[17] 17. Hallegraeff, G.M., Harmful algal blooms: a global overview. Manual on harmful marine microalgae, 2003. 11: p. 25-49.

[18] 18. Hallegraeff, G.M., Ocean Climate Change, Phytoplankton Community Responses, and Harmful Algal Blooms: A Formidable Predictive Challenge. Journal of Phycology, 2010. 46(2): p. 220-235.

[19] 19. Phinn, S., et al., Mapping seagrass species, cover and biomass in shallow waters: An assessment of satellite multi-spectral and airborne hyper-spectral imaging systems in Moreton Bay (Australia). Remote Sensing of Environment, 2008. 112(8): p. 3413-3425. 